\title{
Suprachiasmatic GABAergic Inputs to the Paraventricular Nucleus Control Plasma Glucose Concentrations in the Rat via Sympathetic Innervation of the Liver
}

\author{
Andries Kalsbeek, Susanne La Fleur, Caroline Van Heijningen, and Ruud M. Buijs \\ Netherlands Institute for Brain Research, 1105 AZ Amsterdam, The Netherlands
}

\begin{abstract}
Daily peak plasma glucose concentrations are attained shortly before awakening. Previous experiments indicated an important role for the biological clock, located in the suprachiasmatic nuclei (SCN), in the genesis of this anticipatory rise in plasma glucose concentrations by controlling hepatic glucose production. Here, we show that stimulation of NMDA receptors, or blockade of GABA receptors in the paraventricular nucleus of the hypothalamus (PVN) of conscious rats, caused a pronounced increase in plasma glucose concentrations. The local administration of TTX in brain areas afferent to the PVN revealed that an important part of the inhibitory inputs to the PVN was derived from the SCN. Using a transneuronal viral-tracing technique, we showed that the SCN is connected to the liver via both branches of the autonomic nervous system (ANS). The combination of a blockade of GABA receptors in the PVN with selective removal of either the sympathetic or parasympathetic branch of the hepatic ANS innervation showed that hyperglycemia produced by PVN stimulation was primarily attributable to an activation of the sympathetic input to the liver. We propose that the daily rise in plasma glucose concentrations is caused by an SCN-mediated withdrawal of GABAergic inputs to sympathetic preautonomic neurons in the PVN, resulting in an increased hepatic glucose production. The remarkable resemblance of the presently proposed control mechanism to that described previously for the control of daily melatonin rhythm suggests that the GABAergic control of sympathetic preautonomic neurons in the PVN is an important pathway for the SCN to control peripheral physiology.
\end{abstract}

Key words: autonomic; circadian; glycogen; hypothalamus; paraventricular; microdialysis

\section{Introduction}

Endocrine mechanisms involved in controlling carbohydrate homeostasis, such as pancreatic hormone plasma concentrations and glucose tolerance, exhibit a striking, but primarily unexplained, diurnicity in both humans and rodents (Boden et al., 1996; Van Cauter et al., 1997; La Fleur, 2003). Next to the wellknown hormonal factors, central nervous circuitries may also be involved in the control of hepatic glucose production (HGP) and glucose tolerance (Minokoshi et al., 1999; Obici et al., 2002a). Twenty-four hour rhythms in glucose metabolism are generated by the biological clock, located in the suprachiasmatic nuclei (SCN) (La Fleur et al., 1999, 2001). Data obtained from both human and rat studies strongly suggest that the rise in plasma glucose concentrations at the end of the sleep period is endogenous and caused by increased HGP (Bolli et al., 1984; Boden et al., 1996; La Fleur, 2003). The present study investigated which output mechanisms are used by the SCN to control HGP. HGP can be stimulated by increased glucagon release from the endocrine pancreas, increased activity of the sympathetic input to the liver,

\footnotetext{
Received Dec. 3, 2003; revised June 21, 2004; accepted June 24, 2004.

We thank Wilma Verweij and Bob Baker for correcting our English and Henk Stoffels for making the figures. The expert assistance of J. Timmer in animal husbandry is gratefully acknowledged.

Correspondence should be addressed to A. Kalsbeek, Netherlands Institute for Brain Research, Meibergdreef 33, 1105 AZ Amsterdam, The Netherlands. E-mail: A.Kalsbeek@nih.knaw.nl.

DOI:10.1523/JNEUROSCI.5328-03.2004

Copyright $\odot 2004$ Society for Neuroscience $\quad$ 0270-6474/04/247604-10\$15.00/0
}

or decreased activity of the parasympathetic input to the liver (Shimazu, 1967; Nonogaki, 2000). We hypothesized that the SCN controls HGP via its projections to the autonomic motor neurons that are in control of either the liver or endocrine pancreas (Jansen et al., 1997; Ueyama et al., 1999; La Fleur et al., 2000; Buijs et al., 2001).

The hypothalamic paraventricular nucleus (PVN) is a prominent target area of SCN projections (Watts et al., 1987). In addition to its neuroendocrine function, the PVN also has an important role in coordinating activity within the autonomic nervous system (ANS), as suggested by its connections with preganglionic parasympathetic and sympathetic centers (Saper et al., 1976). Several more-recent experiments have shown direct SCN input to these so-called autonomic PVN neurons (TeclemariamMesbah et al., 1997; Vrang et al., 1997; Cui et al., 2001). The critical role of the PVN in the control of feeding behavior and energy metabolism is well known. Both lesion and (chemical) stimulation experiments have shown its direct involvement in the control of plasma glucose concentrations (Leibowitz et al., 1988; Abe et al., 1989; Gunion et al., 1989; Ionescu et al., 1989).

We began our studies using pseudorabies virus (PRV) injections in the liver of both hepatic parasympathectomized (HPx) and hepatic sympathectomized (HSx) rats. Next, we studied the effect of GABA, NMDA, vasopressinm (VP), and noradrenaline (ant)agonists on plasma glucose concentrations when applied to the PVN. These experiments showed a prominent role of 
GABAergic afferents. We then examined the origin of the GABAergic input to the PVN. Outputs from three different hypothalamic nuclei, well known for their pronounced input to the PVN, were selectively silenced through a temporary arrest of their neuronal activity. Finally, we examined the importance of the autonomic liver innervation for the GABA-mediated control of plasma glucose concentrations.

\section{Materials and Methods \\ Animals}

Male Wistar rats (Harlan Nederland, Horst, The Netherlands) were housed at a room temperature of $21 \pm 1^{\circ} \mathrm{C}$ with a $12 \mathrm{hr}$ light/dark schedule (lights on at 7:00 A.M.). Animals were allowed to adapt to the new environment for 2 weeks before we initiated our studies. Animals were housed four to six per cage until 1 week before surgery, at which time they were transferred to individual cages $(25 \times 25 \times 35 \mathrm{~cm})$. Food and water were available ad libitum, except during experimental sessions, when only water was available. Animals weighed between 300 and $350 \mathrm{gm}$ at the time of the experiments. Rats were anesthetized using a mixture of Hypnorm $(0.05 \mathrm{ml} / 100 \mathrm{gm}$ body weight, i.m.) and Dormicum $(0.04 \mathrm{ml} / 100$ gm body weight, s.c.). During abdominal surgery, the abdominal cavity was bathed regularly with saline to prevent drying of the viscera. The wound was closed with atraumic sutures, and postoperative care was provided with a subcutaneous injection of Fynadine ( $8 \mu \mathrm{l} / 100$ gm body weight). All of the following experiments were conducted under the approval of the Animal Care Committee of the Royal Netherlands Academy of Sciences.

\section{Anatomy}

Fifty animals were used in this part of the study. The rats were divided into the following three groups: $22 \mathrm{HPx}, 20 \mathrm{HSx}$, and 8 hepatic parasympathectomized-sympathectomized (HTx) rats. Immediately after the nerve(s) was cut, $5 \mu$ l of the viral suspension of PRV (Bartha; a generous gift from Dr. C. E. Jacobs, Institute for Animal Science and Health (ID-DLO), Lelystad, The Netherlands) containing $5 \times 10^{6}$ plaque-forming units was injected into the left lobe of the liver. After surgery, the wound was closed with atraumic sutures.

Transneuronal labeling of PRV-Bartha is a well established technique (Ueyama et al., 1999; Buijs et al., 2003) based on the uptake of PRV particles by axon terminals of the neurons projecting to the infected organ, retrograde transport of the virus to the neuronal cell bodies, replication within the neurons, release at the site where the cell bodies or dendrites of the PRV filled neurons are synaptically contacted by axon terminals of other neurons, and subsequent uptake by these axon terminals with retrograde transport. Uptake, but not replication, by glial cells prevents diffusion of the virus to other neurons that do not contact infected neurons (Card, 2001). To determine first-order, second-order, and third-order labeled neurons, different survival times were used. For the first neurons to become visible in the CNS [either in the dorsal motor nucleus of the vagus (DMV) or in the preganglionic neurons in the spinal cord], we called the first-order neurons with neurons labeled immediately after second-order neurons, and so on.

Rats were allowed to survive for 4, 5, or $6 \mathrm{~d}$ and were then deeply anesthetized with sodium pentobarbital and perfused through the left ventricle of the heart with a saline solution, followed by a solution of $4 \%$ paraformaldehyde and $0.05 \%$ glutaraldehyde in PBS (0.1 м PBS, pH 7.2). The brains and spinal cords were then removed and kept overnight in the same fixative. Vibratome $40 \mu \mathrm{m}$ transverse spinal cord and brain sections were washed extensively in Tris-buffered saline (TBS; 0.05 M Trisbuffered saline, $\mathrm{pH}$ 7.4). Every sixth section was incubated overnight in the presence of polyclonal rabbit anti-PRV (anti- $\alpha$ Aujerszky) antibodies (diluted 1:15,000; a generous donation from Dr. C. E. Jacobs). The sections were then incubated for $60 \mathrm{~min}$ in the presence of biotinylated goat anti-rabbit, followed by a $60 \mathrm{~min}$ incubation in the presence of a mixture of Vecta stain ABC kit reagents A and B (Vector Laboratories, Burlingame, CA). The peroxidase antibody complex was visualized by incubating the sections in substrate $\left(0.025 \% 3,3^{\prime}\right.$-diaminobenzidine tetrahydrochloride in TBS supplemented with $0.05 \% \mathrm{H}_{2} \mathrm{O}_{2}$ ).
Table 1. Stereotactic coordinates used for the placement of the microdialysis probes in the different hypothalamic nuclei

\begin{tabular}{llll}
\hline & Anteroposterior & Lateral & Ventral \\
\hline PVN & -1.8 & 2.0 & -7.9 \\
SCN & +1.4 & 1.3 & -8.7 \\
DMH & -3.2 & 1.8 & -8.5 \\
VMH & -2.9 & 2.0 & -9.0 \\
\hline
\end{tabular}

Coordinates are in millimeters for animals with a body weight of $300-350 \mathrm{gm}$. For SCN probe placements, animals were mounted in the stereotact with the toothbar set at $+5.0 \mathrm{~mm}$. For all other probe placements, the toothbar was set at $-3.2 \mathrm{~mm}$.

\section{Surgical procedures}

Hepatic sympathectomy. A laparotomy was performed in the midline. The liver lobes were gently pushed up, and ligaments around the liver lobes were severed to free the bile duct and portal vein complex, which were isolated from each other. At the level of the hepatic portal vein, the hepatic artery breaks up into the hepatic artery proper and the gastroduodenal artery. This division occurs on the ventral surface of the portal vein. At this point, the arteries were separated via blunt dissection from the portal vein. Nerve bundles running along the hepatic artery proper were visualized using a myelin-specific dye (Toluidin Blue) and were removed using microsurgical instruments under an operating microscope $(25 \times$ magnification). Any connective tissue attachments between the hepatic artery and portal vein were also broken, eliminating any possible nerve crossings. To remove the small pieces of nerve tissue from around the artery, a solution of $37 \%$ formaldehyde was applied for $1 \mathrm{~min}$ to the surface of the hepatic artery and portal vein. Despite all of these extra precautions, the percentage of complete hepatic sympathetic denervation was only $50 \%$.

Hepatic parasympathectomy. A laparotomy was performed in the midline. The fascia containing the hepatic branch was stretched by gently moving the stomach and the esophagus. With a myelin-specific dye (Toluidin Blue), the hepatic branch could be revealed as it separates from the left vagal trunk. With the aid of a binocular-operating microscope, the stained neural tissue was transected between the ventral vagus trunk and liver. Also, small blue-stained branches running in the fascia between the stomach and the liver were transected. In the first four rats (see Anatomy), we did not remove these branches and detected remaining unilateral PRV staining in the DMV. With the additional lesioning of these parasympathetic branches running between the stomach and liver, unilateral PRV staining disappeared. Particular care was taken not to damage the dorsal and ventral trunks innervating the stomach and abdominal tissues or blood vessels that run along the hepatic vagus branches.

Jugular venectomy and intracerebral microdialysis probes. Experimental animals destined to undergo infusion and blood-sampling studies were fitted with bilateral microdialysis probes and an intra-atrial silicone catheter through the right jugular vein when their body weight reached 300 gm (Kalsbeek et al., 1996). After surgery, rats were allowed to recover for at least $10 \mathrm{~d}$ to allow complete reinstatement of circadian rhythms (Drijfhout et al., 1995). During experiments, animals were permanently connected to blood-sampling and microdialysis lines, which were attached to a metal collar and kept out of reach from the rats by means of a counterbalanced beam. This allowed all manipulations to be performed outside the cages without handling the animals. The metal collars were attached at least $48 \mathrm{hr}$ before the actual experiment. Animals were handled and sham blood was sampled (i.e., blood was withdrawn and immediately returned) regularly in the week before the first experiment began to familiarize them with all experimental procedures. The microdialysis probes were stereotaxically implanted directly lateral to the PVN, dorsomedial hypothalamic nucleus $(\mathrm{DMH})$, ventromedial hypothalamic nucleus (VMH), or SCN (see Table 1 for stereotaxic coordinates). The loop of the microdialysis probe was positioned in the rostrocaudal direction. Jugular venectomy was performed according to the method of Steffens (1969). We used dental cement to secure the probes and the atrial outlet to two stainless-steel screws inserted into the skull. 


\section{Physiological experiments}

The input port of the dialysis probe was connected to a remote syringe via a fluid swivel (375/22; Instech Laboratories, Plymouth Meeting, PA) interconnected with polyethylene tubing. The syringe contained Ringer's solution with or without experimental drugs. Ringer's perfusion $(3 \mu \mathrm{l} /$ min) was started $2 \mathrm{hr}$ before the start of the $2 \mathrm{hr}$ drug infusion period and lasted for an additional $2 \mathrm{hr}$ after changing back to Ringer's. Dialysates were changed by connecting the polyethylene tubing of the appropriate syringe to the fluid swivel. The dead space between the fluid swivel and the dialysis probe was $45 \mu$ l. Therefore, dialysate-containing drugs reached the dialysis membrane $15 \mathrm{~min}$ after the change of syringes. The 2 $\mathrm{hr}$ drug infusion periods were all initiated at Zeitgeber time (ZT)5, ZT12 being defined as the onset of the dark period. Blood samples $(0.6 \mathrm{ml})$ were taken $5 \mathrm{~min}$ before $(t=0)$ and $5,30,60,120$, and $180 \mathrm{~min}$ after the start of the drug infusion. The following neurotransmitters or (ant)agonists were administered to the PVN: a vasopressin antagonist (VPanta, Manning compound; $50 \mathrm{~mm}$ ), muscimol (MUS; a GABA ${ }_{\mathrm{A}}$ agonist; 100

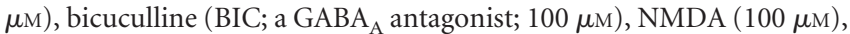
clonidine (CLO; an $\alpha_{2}$-adrenergic agonist; $\left.10 \mu \mathrm{M}\right)$, and isoproterenol (ISO; a $\beta$-adrenergic agonist; $10 \mu \mathrm{M}$ ). The vasopressin antagonist (50 $\mathrm{mM}$ ) was also administered to the DMH. TTX $(1 \mu \mathrm{M})$ was administered to the SCN, PVN, DMH, and VMH according to the same protocol described above. The following three types of control experiments were performed: (1) in one group of animals $(n=8)$, microdialysis probes were perfused with Ringer's; (2) in another group of control animals $(n=28)$, microdialysis probes were deliberately placed 0.5 or $1.0 \mathrm{~mm}$ caudal, lateral, or rostral from the correct PVN coordinates, and 1 week later, these animals were subjected to a BIC infusion; (3) all animals participated in a second experiment 1 week later, with no perfusion of the microdialysis probes and with blood samples taken at the appropriate time points.

\section{Microdialysis probes}

Dialysis probes were constructed according to procedures described previously and used routinely in this laboratory (Kalsbeek et al., 1996). Thin platinum wire $(\phi, 0.05 \mathrm{~mm})$ was inserted into a piece of microdialysis tubing (molecular weight cutoff, $6000 \mathrm{kDa}$ ) and bent; each end was then inserted into one of two pieces of 25 gauge hypodermic tubing that had been soldered together. The dialysis tubing was then secured with epoxy. The U-shaped tip of the microdialysis probe was $1.5 \mathrm{~mm}$ long, $0.7 \mathrm{~mm}$ wide, and $0.2 \mathrm{~mm}$ thick.

\section{Histology}

When the experimental protocol was completed for the nonliver denervated animals, they were anesthetized with $\mathrm{CO}_{2} / \mathrm{O}_{2}$ and decapitated; their brains were removed, blocked, frozen, sectioned $(40 \mu \mathrm{m})$ through the hypothalamus, and stained with cresyl violet. To verify the denervation procedure in liver-denervated animals, we used transneuronal virus tracing with PRV-Bartha as described above. Two days after the second blood-sampling experiment, the viral suspension was injected into the liver, and the rats were allowed to survive for $5 \mathrm{~d}$. These animals were subsequently deeply anesthetized with sodium pentobarbital and perfused through the left ventricle of the heart, and labeled neurons were visualized as described above. If injected PRV-labeled neurons appeared in the spinal cord of HSx rats, they were considered to have experienced a partial hepatic sympathectomy, and these data were excluded from additional analysis. If in HPx rats infected PRV-labeled neurons appeared in the dorsal motor nucleus of the vagus, those animals were considered to have had a partial hepatic parasympathectomy, and these data were excluded from additional analysis.

\section{Analytical methods}

Blood samples were immediately chilled on ice in tubes containing a 10 $\mu l$ solution of $2.5 \%$ EDTA plus $10 \%$ benzamidine hydrochloride and centrifuged at $4^{\circ} \mathrm{C}$. Plasma was then stored at $-80^{\circ} \mathrm{C}$ until additional analysis. Plasma glucose concentrations were determined using a glucose/glucose oxidase-Perid method (Boehringer Mannheim, Mannheim, Germany). Plasma immunoreactive glucagon, insulin, and corticosterone concentrations were measured using radioimmunoassay kits
[LINCO Research (St. Charles, MO) and ICN Biomedicals (Costa Mesa, $\mathrm{CA})$ respectively]; all samples were assayed in duplicate.

\section{Statistical analysis}

We evaluated the kinetics of the plasma concentrations of glucose, glucagon, insulin, and corticosterone as a consequence of the hypothalamic infusion by using two physiological parameters: (1) the increments of their plasma concentrations compared with the $t=0$ value and (2) the areas under the curve (AUC) as a reflection of the overall change in the 180 min postinjection period. The AUC was calculated from the incremental data. All results are expressed as the mean \pm SEM.

The significance of the infusion-induced variations in plasma hormone values was assessed in the following two ways: (1) using a one-way ANOVA with repeated measures [i.e., day (two levels) and sampling (six levels)], and (2) a multivariate ANOVA (MANOVA) using only the data from the first experimental day [sampling (six levels) and, as a betweenanimal factor, treatment (two levels; i.e., drug vs Ringer or intact vs denervated)]. MANOVA was followed by post hoc testing if significant effects of treatment, interaction, or day were detected. Between-group differences in basal hormone concentrations were analyzed using oneway ANOVA and Fischer's least significant difference post hoc tests. Within- and between-group differences in (basal) hormone concentrations were analyzed using the Student's $t$ test for paired and unpaired samples, respectively. Statistical significance was set at $p<0.05$ for a two-tailed test.

\section{Results}

\section{Anatomy}

Virus pattern in HPx rats

In the spinal cord of rats $(n=4)$ with denervation of only the left hepatic parasympathetic branch, PRV-labeled neurons showed up bilaterally in the intermediolateral column (IML) of the thoracic spinal cord. However, PRV-labeled neurons also appeared unilaterally in the DMV of the brainstem. This indicates that not all parasympathetic innervation reaches the liver along this left branch. Therefore, in the following experiments (including the physiological experiments), we also dissected the nerves running over the stomach to the left lobe of the liver as described by Magni and Carobi (1983). This resulted in complete absence of any staining in the DMV in HPx rats. Therefore, infection was limited to first-order neurons in the IML in the thoracic spinal cord of complete HPx rats $(n=4)$ surviving $4 \mathrm{~d}$ after the PRV injection in the liver (Fig. 1B).

HPx rats surviving $5 \mathrm{~d}(n=8)$ had no staining in the DMV (Fig. $1 D$ ) but showed, bilaterally, PRV-labeled cells in the IML in the thoracic spinal cord. In addition to labeling in rats surviving only $4 \mathrm{~d}$, labeling was found in the dorsal horn of the spinal cord (in layers I and IV). In the brainstem, second-order labeled neurons were found in the A5 noradrenergic cell groups, ventrolateral medulla, raphe, and locus ceruleus. Furthermore, secondorder labeling was found in the PVN (Fig. $1 F$ ), zona incerta, lateral hypothalamic area, and retrochiasmatic area of the hypothalamus.

The brains of HPx rats with a survival of $6 \mathrm{~d}(n=4)$ contained third-order neurons in addition to second-order labeled neurons already observed in HPx rats surviving $5 \mathrm{~d}$. In the brainstem, third-order labeled cells were observed in the nucleus of the solitary tract. In the hypothalamus, third-order labeled neurons were observed in areas known to project to the PVN [e.g., medial preoptic area (MPO), anterior hypothalamic area, $\mathrm{DMH}$, arcuate nucleus, $\mathrm{VMH}$, and circumventricular organs]. In the SCN, labeling was present, particularly in the rostral part of the nucleus as well as the dorsomedial and ventral medial parts (Fig. $1 H$ ). HPx rats surviving for $>6 \mathrm{~d}(n=3)$ showed infected cells in a large number of CNS areas. 

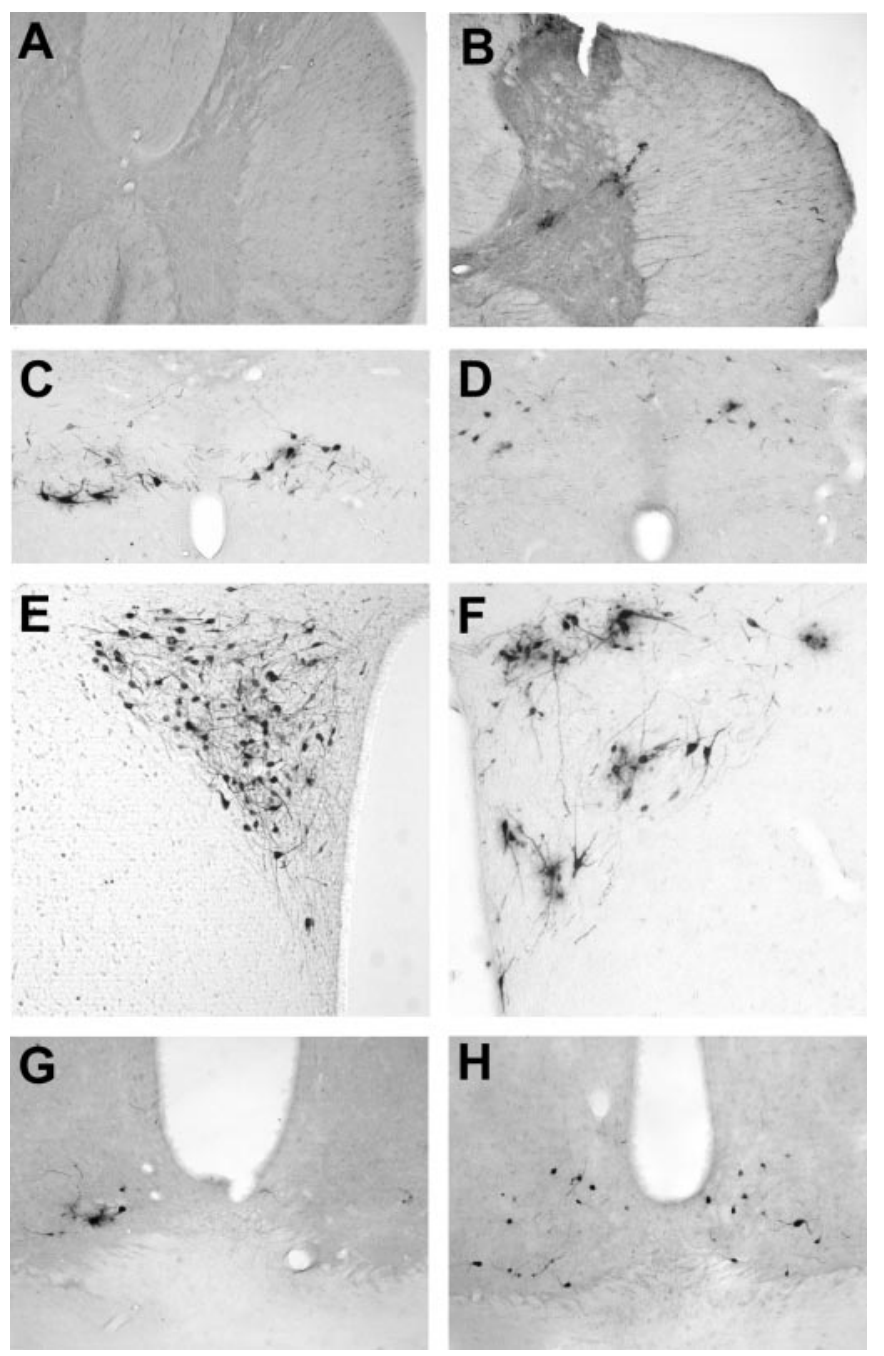

Figure 1. $A-H$, Transversal sections of the spinal $\operatorname{cord}(A, B)$, brainstem $(C, D)$, and hypothalamus $(E-H)$ stained for the transneuronal tracer (PRV). $A, C, E$, and $G$ are sections from $H S x$ rats, and $B$, $D, F$, and $H$ aresections from $H P x$ rats. $A, B$, Sections of the spinal cord at the thoraciclevel. The surviving time after PRV injection in the liver is $4 \mathrm{~d}$. HSx rats do not show first-order labeling in the IML of the thoracic part of the spinal cord $(A)$, whereas first-order PRV labeling is observed in the thoracic part of the IML of the HPx rats $(B) . C, D$, Sections at the level of the brainstem. The survival time after PRV injection in the liver is $5 \mathrm{~d}$ ( $C$ or $6 \mathrm{~d}(D)$. PRV labeling is observed in the DMV of HSx rats ( $($ but not in the DMV of HPx rats $(D)$. $E-H$, Sections at the level of the hypothalamus. The survival time is $6 \mathrm{~d}$. Intense PRV labeling is observed in the PVN of both HSx rats $(E)$ and HPx rats ( $F$ ). PRV-labeled neurons are clearly observed in the $\mathrm{SCN}$ of HSx rats $(G)$ and $\mathrm{HPx}$ rats $(H)$.

\section{Virus pattern in HSx rats}

Fifteen of 24 HSx rats experienced a complete sympathetic denervation of the liver, according to the labeling pattern of PRV in the spinal cord, brainstem, and hypothalamus. HSx rats $(n=3)$ surviving $4 \mathrm{~d}$ showed first-order labeled neurons bilaterally in the DMV (Fig. 1C). No staining was detected in the spinal cord, demonstrating evidence for complete hepatic sympathectomy (Fig. $1 A$ ). In the brain of HSx rats $(n=7)$ surviving $5 \mathrm{~d}$, secondorder labeled cells appeared in many cell groups in the brainstem, hypothalamus, and limbic structures, such as in the PVN (Fig. $1 E), \mathrm{DMH}$, and MPO. These are all nuclei known to receive a projection from the SCN. In HSx rats surviving $6 \mathrm{~d}(n=5)$, additional staining (i.e., third-order labeling) was observed in the SCN (Fig. 1G), with a labeling pattern similar to that seen in the SCN of HPx rats, and in the VMH.
Virus pattern in HTx rats

After total hepatic denervation, PRV injection into the liver resulted in no PRV-positive staining in the CNS in the three rats surviving for $6 \mathrm{~d}$ after injection.

\section{Physiology}

One hundred and seventy-five animals were used in this part of the study. Approximately $15 \%$ of the data had to be discarded because of incorrect probe placements, incomplete denervation, or an incomplete data set. Histological examination of brain tissue revealed that in the majority of the animals, the tip of the microdialysis probes was consistently lowered to within 50-100 $\mu \mathrm{m}$ of the dorsal or lateral border of the different hypothalamic nuclei selected (Fig. 2). In addition, this figure illustrates the limited damage inflicted by the microdialysis technique.

\section{PVN infusions}

Of the six different drugs administered to the PVN, only three produced a significant deviation of plasma glucose concentrations compared with those found during control conditions, according to ANOVA (i.e., BIC, NMDA, and the VP antagonist). BIC administration significantly increased plasma glucose concentrations compared with the control day or the Ringer-infused group (supplemental material, available at www.jneurosci.org). Administration of NMDA also caused significant hyperglycemia compared with the control day and Ringer-infused animals. The effects of the VP antagonist were less pronounced (i.e., only a significant interaction effect) (supplemental material, available at www.jneurosci.org). Also, the time course of the VP antagonistproduced hyperglycemia differed from that of BIC and NMDA (Fig. 3). BIC and NMDA caused a significant elevation of plasma glucose concentrations during most of its application to the PVN ( $t=30-120)$, whereas the VP antagonist only caused a significant increase at $t=120$ and $t=180$. The effect of the VP antagonist was specific for the PVN, because administration to the DMH did not affect plasma glucose concentrations (Fig. 4) (supplemental material, available at www.jneurosci.org).

In contrast, all drugs except NMDA significantly affected plasma corticosterone concentrations (effects of day and treatment for all five drugs, $p \leq 0.05$ ) (supplemental material, available at www.jneurosci.org). BIC and both noradrenaline agonists caused an immediate and pronounced increase in plasma corticosterone concentrations, whereas the VP antagonist (either in the PVN or DMH) caused a more moderate increase 30 and 60 min after the start of its application. During application of the GABA-agonist MUS, plasma corticosterone concentrations remained basal, but a clear rebound effect occurred after application, resulting in increased plasma corticosterone concentrations only at $t=180$. Administration of Ringer or NMDA did not cause significant changes in the plasma corticosterone concentrations. In Figure 5, the glucose and corticosterone changes, as caused by the administration of the different drugs to the PVN, are compared. It is evident that there is no clear correlation between the corticosterone and glucose responses. BIC administration caused a significant increase in both plasma glucose and plasma corticosterone concentrations, NMDA caused hyperglycemia but no increased release of corticosterone, and the two adrenergic agonists significantly affected corticosterone release but not plasma glucose concentrations. During ISO administration, a pronounced decrease in plasma glucose concentrations was observed, although not significantly different from the control experiments. Because this decrease was not accompanied by significant changes in plasma insulin or plasma glucagon concentrations, it 
was most likely attributable to the relatively high starting concentrations of plasma glucose in this particular group (supplemental material, available at www.jneurosci.org).

The pronounced effect of BIC on plasma glucose concentrations was accompanied by a significant increase in plasma glucagon concentrations (sampling, $p=0.358$; day, $p=0.081$; interaction, $p=0.029$ ), whereas plasma insulin concentrations were not significantly affected (sampling, $p=0.973$; day, $p=$ 0.908 ; interaction, $p=0.377$ ). Administration of MUS into the PVN affected neither plasma glucagon concentrations (sampling, $p=0.636$; day, $p=0.445$; interaction, $p=0.566$ ) nor plasma insulin concentrations (sampling, $p=0.465$; day, $p=0.361$; interaction, $p=0.280)$.

The basal plasma glucose and corticosterone concentrations (i.e., before the administration of the different drugs into the PVN) are displayed in supplemental Table 2 (available at www.jneurosci.org as supplemental material). In general, their basal concentrations in experimental and control sessions did not differ, varying between 6.0 and $7.5 \mathrm{mmol} / \mathrm{l}$. Also, basal plasma glucagon (supplemental material, available at ww jneurosci.org) and plasma insulin concentrations of the experimental and control days were comparable before the administration of BIC and MUS (insulin [BIC], $2.5 \pm 0.3 \mathrm{vs}$ $1.9 \pm 0.3$; [MUS], $2.1 \pm 0.2$ vs $3.0 \pm 0.6$ for experimental vs control days).

\section{Silencing PVN inputs by TTX infusions}

To identify the main source of the GABAergic input to the PVN responsible for the increased plasma glucose concentrations after administration of BIC, we selectively silenced three major afferent inputs to the PVN by local administration of the sodium channel blocker TTX. In addition, TTX was also administered to the PVN itself. Infusion of TTX significantly increased plasma corticosterone concentrations (i.e., significant effects of drug and/or interaction) when administered into the VMH, DMH, or SCN but not into the PVN (supplemental material, available at www.jneurosci.org). With regard to plasma glucose concentrations, only TTX infusions aimed at the $\mathrm{DMH}$ and SCN caused a significant increase in plasma glucose concentrations (Fig. 6) (supplemental material, available at www. jneurosci.org). TTX administration into the VMH and PVN tended to decrease plasma glucose concentrations. However, the decrease only reached significance in the VMH group (effect of sampling, $p=0.030$ ), but it was not specific to TTX, because plasma glucose levels also decreased during the control day. Thus, no significant effects of day or interaction were found. Indeed, plasma glucose concentrations slowly decreased during nonstimulated conditions, because the four control experiments Student's $t$ tests.
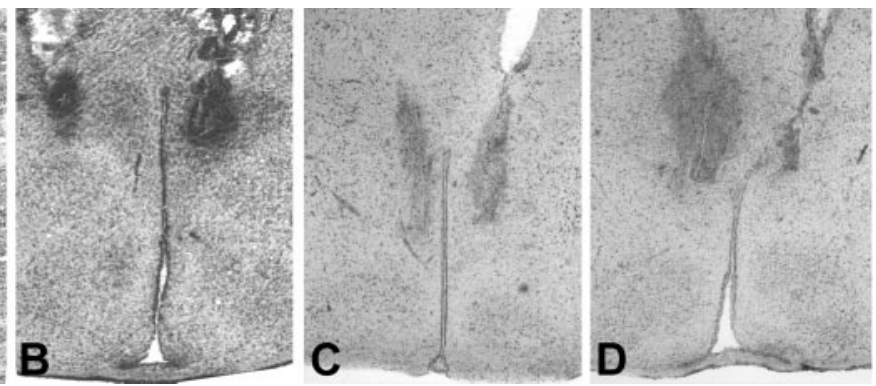

Figure 2. $A-D$, Photomicrographs illustrating the bilateral placement of microdialysis probes in the $\operatorname{PVN}(A, B)$ and $D M H(C, D)$. Probe placements in four different animals are shown to illustrate the limited variation in probe placement. Moreover, these sections also illustrate clearly that the tissue damage caused by the placement of microdialysis probes is only limited.
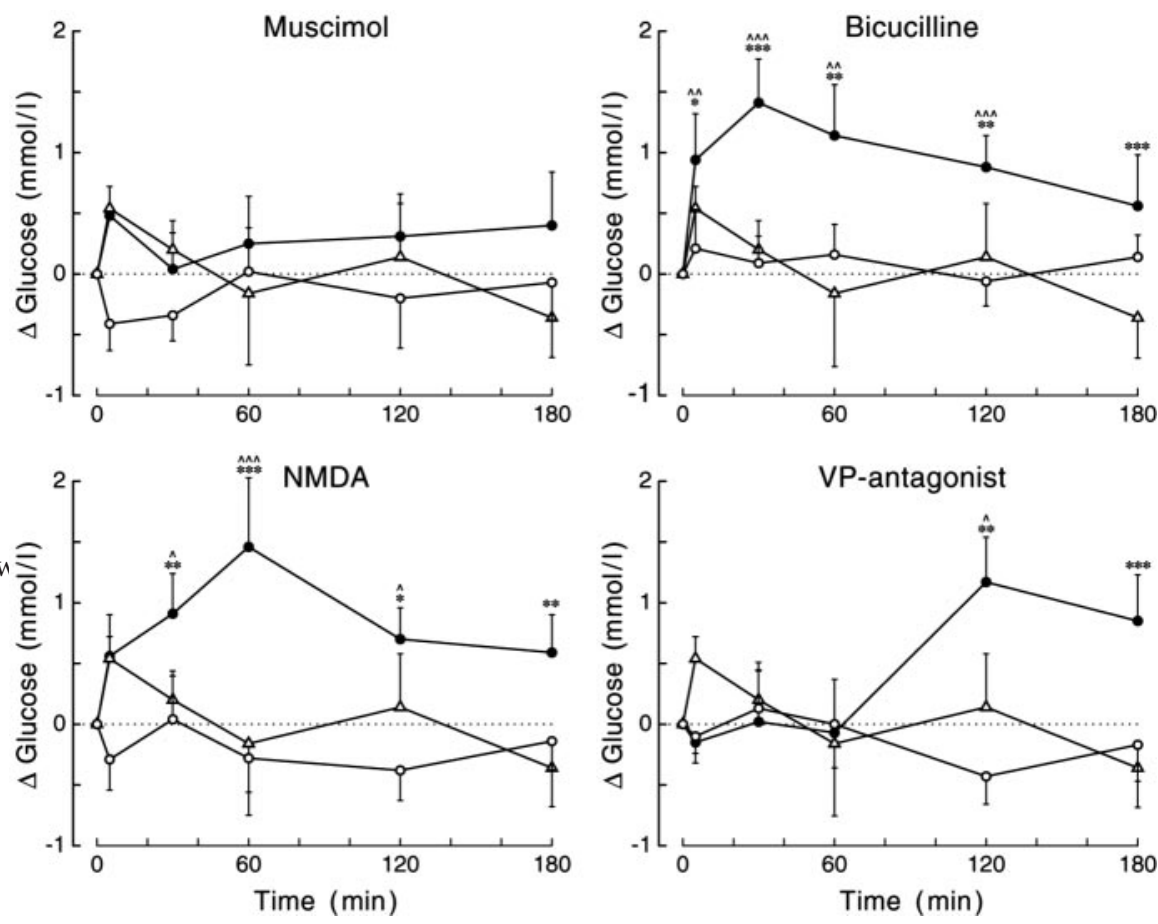

Figure 3. Changes in plasma glucose concentrations during a $2 \mathrm{hr}$ administration of bicuculline, muscimol, NMDA, or VP antagonist in the PVN. Filled symbols indicate the effect of the drug, whereas open symbols show the result of the control experiment in the same group of animals (circles) or the effect of Ringer administration in a separate group of animals (triangles). All drugs except MUS caused a significant increase in plasma glucose concentrations. MANOVA indicated significant effects of day (i.e., experimental vs control) and treatment (i.e., drug vs Ringer) for both BIC and NMDA ( $p=0.001$ and $p=0.004$, and $p=$ 0.002 and $p=0.012$, respectively). Moreover, all three drugs showed significant interaction effects of both sampling versus day and sampling versus treatment. For basal $(t=0)$ concentrations and additional statistical details, see supplemental Tables 1 and 2 (available at www.jneurosci.org as supplemental material), respectively. ${ }^{* \wedge} p<0.1,{ }^{* *, \wedge \wedge} p<0.05$, and ${ }^{* * *, \wedge \wedge \wedge} p<0.01$, respectively. Symbols indicate the results of paired $(\wedge)$ (i.e., experimental vs control) and unpaired $(*)$ (i.e., drug vs Ringer)

$(n=34)$ showed a significant decrease in plasma glucose concentrations (effect of sampling, $p=0.015$ ). TTX infusion in the PVN itself, or in one of its three input areas, did not significantly affect plasma glucagon (supplemental material, available at www.jneurosci.org) or plasma insulin concentrations (data not shown). Basal plasma glucose, glucagon, and corticosterone concentrations are displayed in the supplemental material (available at www.jneurosci.org).

\section{Liver denervation}

To investigate which output pathway of the PVN was responsible for the increased plasma glucose concentrations after PVN administration of BIC (i.e., increased glucagon release by the pan- 

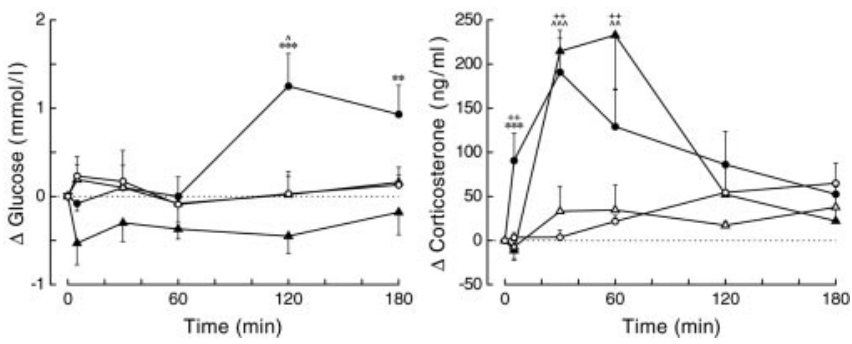

Figure 4. Changes in plasma glucose and plasma corticosterone concentrations as a result of VP antagonist administration in either the PVN (circles) or DMH (triangles). Despite the comparable responses of plasma corticosterone ( $p=0.621$ and $p=0.047$ for group and group $\times$ sample effects, respectively), the effects of the VP antagonist on plasma glucose are clearly dissimilar ( $p=0.011$ and $p=0.005$ for effects of group and group $\times$ sample, respectively). Closed symbols represent the effect of the VP antagonist, and open symbols represent results of the control experiment. $\wedge p<0.1,{ }^{* * \wedge \wedge,++} p<0.05,{ }^{* * *, \wedge \wedge \wedge} p<0.01$. The asterisks indicate the results of the unpaired (i.e., PVN vs DMH) Student's $t$ tests. ${ }^{\wedge}$ and ${ }^{+}$indicate the results of paired (i.e., experimental day vs control day) Student's $t$ tests for the PVN $\left({ }^{\wedge}\right)$ and DMH $\left({ }^{+}\right)$, respectively.

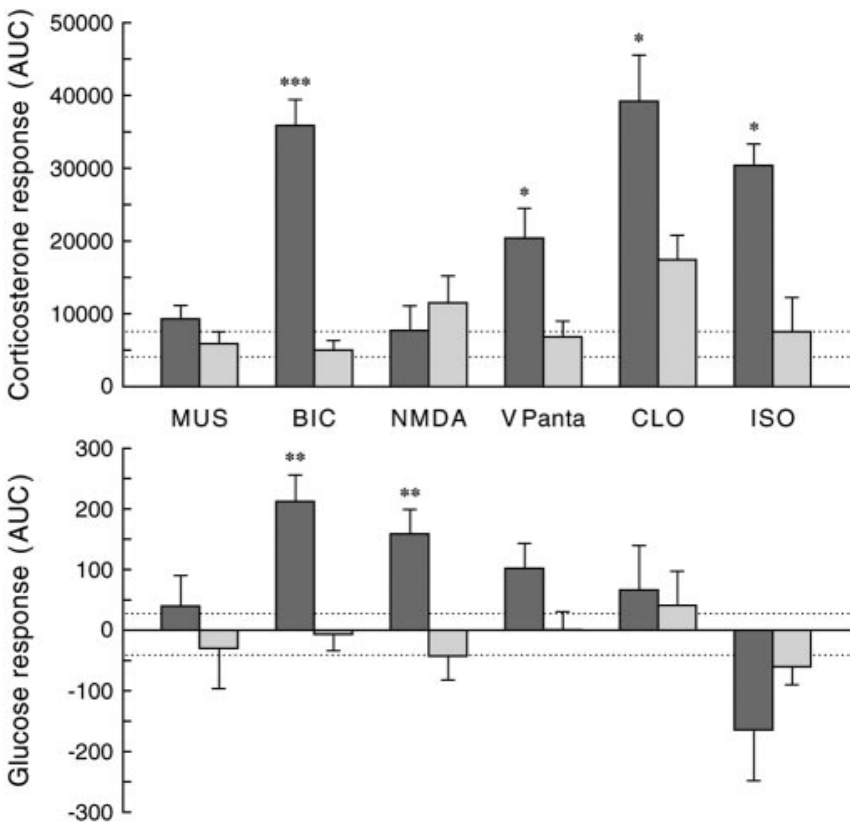

Figure 5. Differential plasma corticosterone and plasma glucose responses as a consequence of a $2 \mathrm{hr}$ administration of MUS, BIC, NMDA, VP antagonist (VPanta), CLO, or ISO in the PVN. Corticosterone and glucose responses are expressed as area under the curve to reflect the overall change in the $3 \mathrm{hr}$ sampling period. The AUC was calculated from the incremental data for each individual animal. The dark bars indicate the integrated response (AUC) during drug administration, and the light bars indicate the response during the control experiment 1 week later. Dashed lines indicate the higher and lower limits (i.e., mean \pm SEM) of the corticosterone and glucose response during the administration of Ringer into the PVN. ${ }^{*} p<0.05,{ }^{* *} p<0.01$, ${ }^{* * *} p<0.001$ according to paired Student's $t$ tests.

creas or a changed autonomic input to the liver), we repeated the BIC administration into the PVN in animals with a selective denervation of either the sympathetic or parasympathetic input to the liver. Viral tracing revealed that of the 40 rats on which we operated, 11 had to be discarded because of an incomplete denervation procedure. Basal concentrations of plasma glucose, corticosterone, glucagon, and insulin were not changed in any consistent way by the obstruction of the autonomic input to the liver (supplemental material, available at www.jneurosci.org). ANOVA only indicated a significant difference in the basal plasma glucose concentrations during control days. Post hoc test- ing showed that plasma glucose concentrations in liver-intact animals were higher than in both denervated groups. However, this effect was only observed during the control sessions and not 1 week earlier, during the experimental days.

The stimulatory effect of BIC administration in the PVN on plasma glucose concentrations could only be reproduced in the HPx animals. The hyperglycemic response in these animals did not differ from that in intact animals (Fig. 7) (supplemental material, available at www.jneurosci.org). In contrast, a hyperglycemic effect of BIC was completely absent in the HSx animals. In fact, post hoc analysis revealed that the interaction effect ( $p=$ 0.017 ) was caused by significant decreases in plasma glucose concentrations at $t=5$ and $t=180(p=0.015$ and $p=0.050$, respectively) in the HSx animals. Consequently, the plasma glucose response in HSx but not in HPx animals differed significantly from the response seen in intact animals (supplemental material, available at www.jneurosci.org).

As with plasma glucose, HPx did not effect the plasma glucagon increase in response to BIC administration in the PVN (Fig. $7)$. In contrast, mean glucagon concentrations in HSx animals were decreased (Fig. 7). However, in all three treatment groups, the glucagon response during BIC administration differed significantly from that during control days (i.e., significant effects of day and/or interaction) (supplemental material, available at www.jneurosci.org). Also, in neither of the two liver-denervated groups did the glucagon response differ significantly from that in the liver-intact controls (i.e., no significant effects of group or interaction).

In contrast, hepatic denervation significantly affected the plasma corticosterone responses. A significantly higher corticosterone response was observed in both HPx and HSx animals during BIC administration to the PVN (supplemental material, available at www.jneurosci.org), and AUC increased by almost 50\%.

MUS administration in the PVN of $\operatorname{HPx}(n=7)$ and $\mathrm{HSx}(n=$ 7) animals did not effect plasma glucose, glucagon, or corticosterone concentrations in any way other than observed for the liverintact animals (data not shown).

\section{Specificity of drug administration by microdialysis}

In addition to correct probe placement, spread of the administered drugs is always an important concern for the present type of studies. The tissue distribution of administered drugs is often estimated by the coadministration of a colored dye. However, because of differences in molecular size and properties, it is likely that although the distribution of the dye and the drug overlap, they will not be identical. Moreover, the effective spread of a drug will also depend on its effective concentration. Therefore, we chose to use a functional parameter to estimate the spread of the different drugs administered. A two-way ANOVA comparing the effect of TTX in the four different brain areas studied revealed a significant effect of brain area for both plasma glucose $(p=$ 0.014 ) and corticosterone ( $p=0.037)$ concentrations. By comparing the infusion effects of TTX and the vasopressin antagonist in three pairs of neighboring brain nuclei (Table 2), it could be calculated that the effective radius of the TTX diffusion is $<0.6$ $\mathrm{mm}$. In addition, in Figure 8, we compared the effect of BIC administration in the PVN with the deliberate administration of BIC outside the PVN. Twenty-four of the 28 animals operated on produced a complete data set. PVN probe placements were divided into the following groups: those close to or within the PVN $(n=7)$, those within $1.0 \mathrm{~mm}$ of its caudal border $(n=7)$, those within $1.0 \mathrm{~mm}$ of its lateral or rostral borders $(n=5)$, and those $>1 \mathrm{~mm}$ away from the caudal border of the PVN. When BIC was 
applied in the surroundings of the PVN but within $1 \mathrm{~mm}$ of its borders, the hyperglycemic effect of BIC was significantly delayed compared with administrations precisely within the PVN. When applied $>1$ $\mathrm{mm}$ distant from the PVN, no significant effects on plasma glucose could be detected. The results of groups $\mathrm{C} 1$ and $\mathrm{D}$ clearly show that the hyperglycemic effect of BIC was attributable to its effect on (preautonomic) PVN, and not DMH, neurons. Moreover, the present results are also in accordance with our previous data showing that, only when applied close to the PVN, BIC was able to prevent the lightinduced inhibition of melatonin release (Kalsbeek et al., 2000). The differentiation of the infusion effects, when the same drug is administered to different hypothalamic nuclei or $>0.5 \mathrm{~mm}$ outside the destined nucleus, is clearly consistent with the hypothesis that the BIC, VP antagonist, and TTX data are indeed mediated by the brain areas that were targeted.

\section{Discussion}

The present study shows that the SCN is connected to the liver through both the parasympathetic and sympathetic branches of the ANS via preautonomic neurons located in, among others, the PVN. In addition, activation of PVN neurons, either by stimulation of NMDA receptors or by relief from inhibitory GABAergic inputs, resulted in a pronounced increase in plasma glucose concentrations. The increased plasma glucose concentrations are most likely attributable to an increased HGP, caused by activation of sympathetic preautonomic neurons in the PVN, because selective denervation of the sympathetic but not the parasympathetic, input to the liver completely prevented the BIC-induced hyperglycemia. The hyperglycemic effect of GABA blockade in the PVN was partially mimicked by silencing the activity of SCN or DMH but not of VMH neurons. We propose that the daily rise in basal plasma glucose concentrations at dusk is caused by a biological clock-mediated withdrawal of the GABAergic inhibition of sympathetic preautonomic PVN neurons. The presently identified hypothalamic pathway provides a neuroanatomical substrate not only for the biological clock control of daily glucose homeostasis but also for the recently reported central feedback actions of peripheral hormones (Kamohara et al., 1997; Obici et al., 2002a,b).

The combination of transneuronal virus tracing, specific hepatic denervation, and carefully timed periods of survival enabled us to provide a detailed scheme of the neural pathways by which the SCN can influence the liver. Third-order SCN labeling was found in both the HSx and HPx rats, indicating that the SCN is able to provide signals to the liver through both branches of the ANS, probably even by different populations of SCN neurons (Buijs et al., 2003). Again, in HSx rats, many hypothalamic cell groups project to the DMV, whereas in HPx rats, the PVN was the only SCN target area showing second-order labeling (Buijs et al., 2001).

The pronounced rhythm in liver glycogen content, with its acrophase at light onset, has been shown to be attributable to daily changes in the balance between the activities of glycogen-
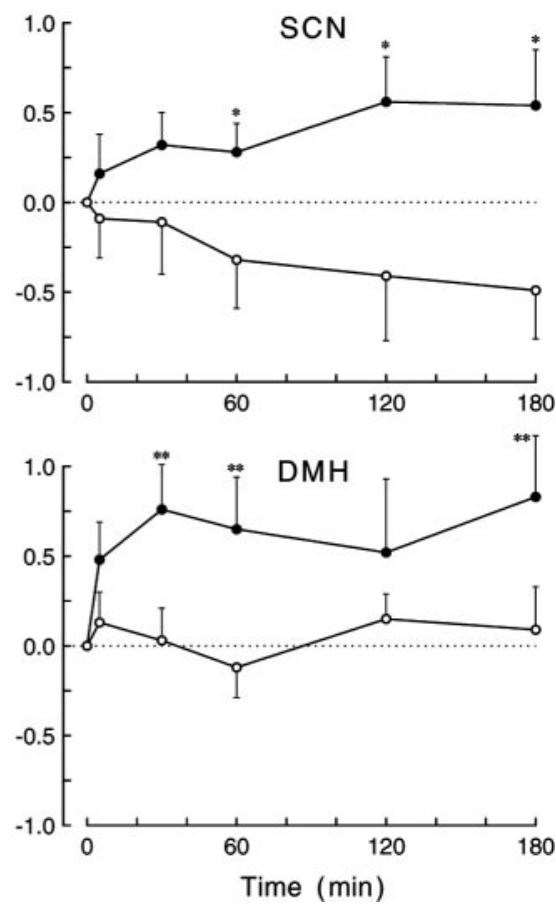

$\mathrm{VMH}$

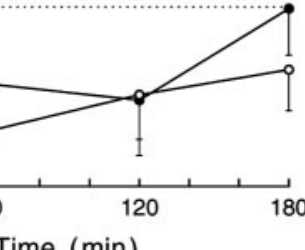



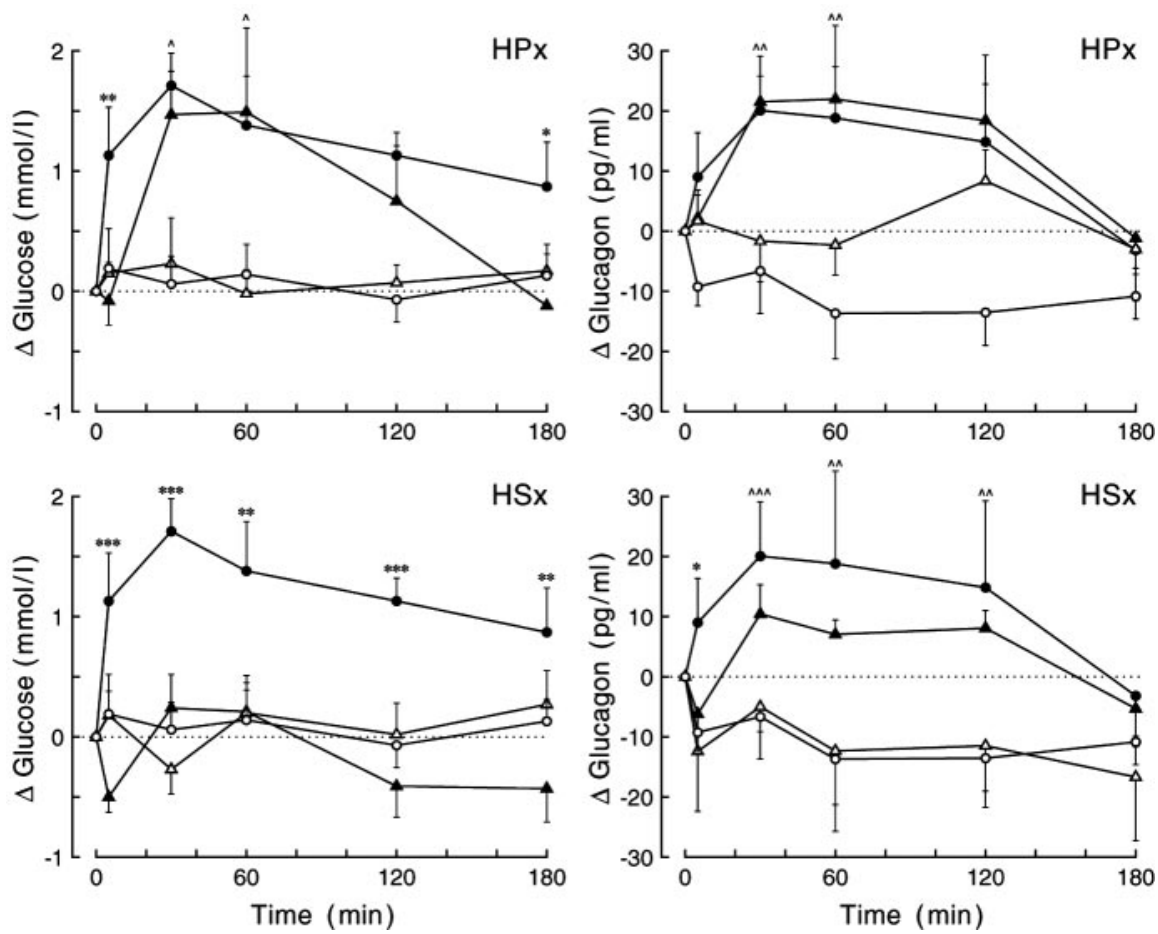

Figure 7. Changes in plasma glucose (left panels) and plasma glucagon (right panels) concentrations during the $2 \mathrm{hr}$ administration of BIC in the PVN of liver-intact and liver-denervated animals. Data from the HPx and HSx liver-denervated animals are displayed in the top and bottom rows, respectively. In both intact and liver-denervated animals, all BIC-induced glucose and glucagon responses were significantly increased compared with the respective control experiments $(p<0.03$ for all day $\times$ sampling interactions), except for the glucose response in HSx animals (bottom left). Moreover, only the glucose response in the HSx group differed significantly from that in liver-intact animals $(p<0.001$ and $p=0.016$ for group and group $\times$ sampling effects, respectively). In neither of the two liver-denervated groups did the glucagon response differ from that in liver-intact animals ( $p>0.5$ for both group and group $\times$ sampling effects). For basal $t=0$ concentrations and additional statistics, see supplemental Tables 5 and 6 (available at www.jneurosci.org as supplemental material), respectively. Closed symbols indicate the effect of BIC, and open symbols show the results of the respective control experiments. Circles, Liver-intact animals; triangles, liver-denervated animals. ${ }^{*, \wedge} p<0.1,{ }^{* *, \wedge \wedge} p<0.05$, and ${ }^{* * *, \wedge \wedge \wedge} p<0.01$, respectively. ${ }^{\wedge}$ and ${ }^{*}$ indicate the results of paired (i.e., experimental day vs control day) Student's $t$ tests in the liver-denervated animals and unpaired (i.e., liver-intact animals vs denervated animals on experimental days) Student's $t$ tests, respectively.

Table 2. Brain region-specific effects of TTX and VP antagonist administration on plasma glucose and plasma corticosterone concentrations

\begin{tabular}{|c|c|c|c|c|}
\hline & $\begin{array}{l}\text { Radial } \\
\text { distance }^{a}\end{array}$ & & TTX & VP antagonist \\
\hline SCN-PVN & $1.8 \mathrm{~mm}$ & $\begin{array}{l}\text { Brain area } \\
\text { Interaction }\end{array}$ & $\begin{array}{l}p=0.024 \text { (glucose) } \\
p=0.088\end{array}$ & \\
\hline PVN-DMH & $1.5 \mathrm{~mm}$ & $\begin{array}{l}\text { Brain area } \\
\text { Interaction }\end{array}$ & $\begin{array}{l}p=0.009 \text { (glucose) } \\
p=0.024\end{array}$ & $\begin{array}{l}p=0.005 \text { (glucose) } \\
p=0.011\end{array}$ \\
\hline $\mathrm{DMH}-\mathrm{VMH}^{b}$ & $0.6 \mathrm{~mm}$ & $\begin{array}{l}\text { Brain area } \\
\text { Interaction }\end{array}$ & $\begin{array}{l}p=0.055 \text { (glucose) } \\
p=0.039\end{array}$ & $\begin{array}{l}p=0.408 \text { (corticosterone) } \\
p=0.006\end{array}$ \\
\hline
\end{tabular}

$p$ values indicate the result of a two-way ANOVA with time as the within-animal factor and brain area as the between-animal factor.

${ }^{a}$ The radial distance is calculated from the difference between the stereotactic coordinates shown in Table 1. SCN coordinates were adapted to the flat skull position as used for the other brain areas.

${ }^{b}$ The corticosterone data for the DMH-VMH comparison are derived from the study by Kalsbeek et al. (1996).

hormonal changes as the primary cause for BIC-induced hyperglycemia (Nonogaki et al., 1994; Cole and Sawchenko, 2002).

The hypothalamic areas for TTX infusion were selected on the basis of their known projections to the PVN (Luiten and Room, 1980; Boudaba et al., 1996) and the presence of third-order labeling after virus injection in the liver. Silencing of either the SCN or DMH input caused an increase in plasma glucose concentrations, although to a lesser extent than those observed when all of the GABAergic inputs to the PVN were silenced. The absence of a clear effect of VMH-TTX on plasma glucose concentrations substantiates the observation that GABAergic neurons are virtually absent from the VMH (Okamura et al., 1990) and that stimulation instead of inhibition of $\mathrm{VMH}$ neurons leads to hyperglycemia (Narita et al., 1994). GABAergic SCN inputs to the autonomic PVN neurons have been demonstrated in a number of electrophysiological experiments (Hermes et al., 1996; Cui et al., 2001; Wang et al., 2003). In addition, there is also evidence for GABAergic projections from the DMH to the PVN (Boudaba et al., 1996). Because the DMH is also a target area for SCN projections (Watts et al., 1987; Buijs et al., 1993), the SCN might use both direct and indirect GABAergic projections to the preautonomic neurons in the PVN to control basal plasma glucose concentrations, implying that SCN projections to the GABAergic DMH neurons are stimulatory. In addition to the SCN and $\mathrm{DMH}, \mathrm{GABAergic}$ inputs from other sources may restrain the activity of preautonomic PVN neurons controlling HGP. For instance, both the arcuate nucleus and preoptic area show third-order virus labeling, send GABAergic projections to the PVN (Roland and Sawchenko, 1993; Boudaba et al., 1996), and are involved in glucose homeostasis (Zaia et al., 1997; Choi and Dallman, 1999; Ferreira et al., 1999). Interestingly, the preoptic area and arcuate nucleus are also targets for SCN projections. In contrast, GABAergic projections from these areas could also be implicated in the transmission of information other than circadian to the liverdedicated PVN neurons. For instance, leptin receptor-containing neurons in DMH and arcuate nucleus might be involved in the hyperglycemic effect of intracerebroventricular administration of leptin (Kamohara et al., 1997), whereas projections from the preoptic area seem to be involved in the increased HGP during cold exposure (Ferreira et al., 1999).

It seems logical to assume an involvement of the vasopressinergic SCN projection to the intermediate GABA neurons in the subPVN (Hermes et al., 2000) in the hyperglycemic effect of the vasopressin antagonist. However, it is not clear why the VP antagonist-induced hyperglycemia is so much delayed compared with its stimulatory effect on corticosterone release (also operating via the intermediate GABA neurons). Moreover, administration of the VP antagonist into the DMH did not cause hyperglycemia, but the increase in plasma corticosterone concentrations was similar to that seen after administration in the PVN (Fig. 4). Application of NMDA into the PVN did not activate the hypothalamic-pituitary-adrenal axis (Fig. 3), probably because it stimulated the GABAergic interneurons in the subPVN, which subsequently inhibited the hypophysiotropic corticotropinreleasing hormone-containing neurons in the PVN (Cole and Sawchenko, 2002). However, NMDA administration in the PVN did cause hyperglycemia, indicating that GABAergic neurons in the subPVN project to the endocrine but not the 


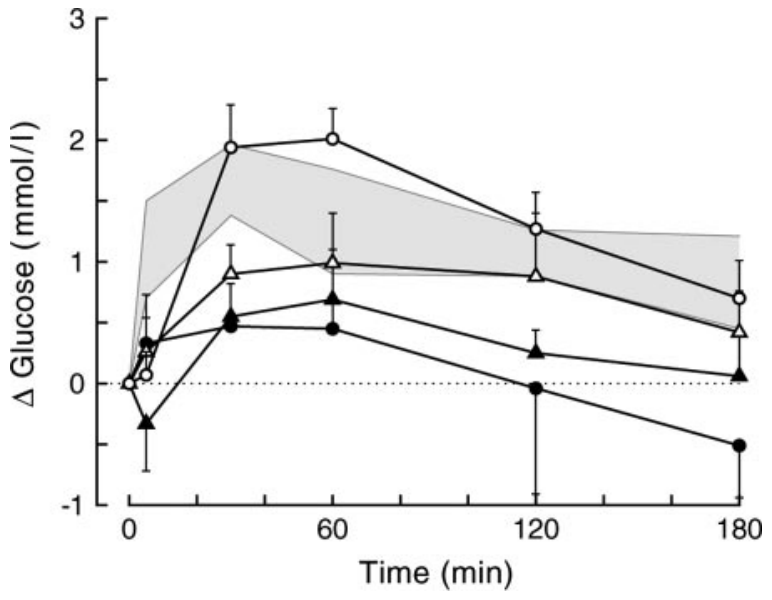

Figure 8. Plasma glucose changes during BIC administration into or outside the PVN of liver-intact animals. Five different treatment groups are displayed. The gray shading indicates the mean \pm SEM from the original group of PVN infusions (group A). Probe placements for the additional control group are divided in those within or touching the PVN (group B; open circles; $n=7$ ), those within $<1 \mathrm{~mm}$ of the caudal borders of the PVN (group (1; open triangles; $n=$ 7 ), those within $<1 \mathrm{~mm}$ of the lateral or rostral borders of the PVN (group C 2 ; closed triangles; $n=5$ ), and those $>1 \mathrm{~mm}$ away from the caudal border of the PVN (group D; closed circles; $n=$ 5). Depending on whether groups $C 1$ and $C 2$ were separated or combined, the MANOVA for the overall data showed significant effects of group ( $p=0.023$ and $p=0.019$, respectively) and group $\times$ time ( $p=0.092$ and $p=0.018$, respectively). Comparing the different groups displaying probe placements outside the PVN (i.e., $C 1, C 2, D)$ with the within-PVN placement group (i.e., B) revealed significant group effects for groups $C 2$ and $D(p=0.003$ and $p=0.008$, respectively) and significant group $\times$ time effects for groups $C 1, C 2$, and $D(p=0.035, p=$ 0.024 , and $p=0.052$, respectively). When compared with the original within-PVN group (i.e., the gray area), significant effects of group were found for groups ( and $D$ ( $p=0.033$ and $p=$ 0.007 , respectively), and a significant group $\times$ time effect was found for group $B(p=0.045)$. Separating group C in caudal (C1) and lateral plus rostral (C2) placements revealed that only group C2 differed significantly from group A ( $p=0.004$ for the effect of group). In all groups except for groups $C 2$ and $D$, the within-animal comparison showed significant effects of day and day $X$ time (i.e., the BIC day differs significantly from the control day).

autonomic PVN neurons. Interestingly, administration of vasopressin into the PVN is unable to inhibit melatonin release (Kalsbeek et al., 2000). Therefore, it appears that endocrine (or hypophysiotropic) neurons, but not autonomic neurons, in the PVN are sensitive to the (indirect) effects of vasopressin. However, this leaves the hyperglycemic effect of the vasopressin antagonist still unexplained. Previously, it has been shown that vasopressin antagonists applied in the supraoptic nucleus (SON) will increase the electrical firing rate of the (vasopressinergic) SON neurons (Ludwig and Leng, 1997). The increased concentrations of plasma vasopressin that are therefore likely to result from administration of a VP antagonist into the PVN (but not the DMH) will result in increased gluconeogenesis by a direct effect on the liver (Hems et al., 1978). Indeed, the delayed increase in plasma glucose concentrations fits better with an effect on gluconeogenesis than on glycogenolysis.

Previously, we provided physiological evidence for a daily rhythm in the amount of GABA that was released from SCN terminals in the vicinity of pineal-dedicated PVN neurons (Kalsbeek et al., 1999, 2000), with a peak release of GABA during the light period. The present data indicate that a similar mechanism could be operative for the control of the daily rhythm in plasma glucose. A gradual biological clock-mediated withdrawal of GABAergic inputs to preautonomic PVN neurons during the light period results in a changing balance of the autonomic input to the liver (i.e., switching from a parasympathetic stimulation of glucose uptake and glycogenesis at dawn to a sympathetic stimulation of glycogenolysis and glucose production at dusk) and is responsible for the daily glucose peak at dusk. Future studies will have to show how the SCN (via the sympathetic and parasympathetic branches of the ANS) controls the balance between glycogenolysis and glycogenesis. In this regard, glutamatergic SCN outputs might also be important (Csaki et al., 2000; Chen et al., 2003; Perreau-Lenz et al., 2004). Indeed, apart from GABA, glutamatergic (SCN) inputs to the PVN may also show a daily variation.

\section{References}

Abe M, Saito M, Shimazu T (1989) Neuropeptide Y and norepinephrine injected into the paraventricular nucleus of the hypothalamus activate endocrine pancreas. Biomed Res 10:431-436.

Boden G, Chen X, Urbain JL (1996) Evidence for a circadian rhythm of insulin sensitivity in patients with NIDDM caused by cyclic changes in hepatic glucose production. Diabetes 45:1044-1050.

Bolli GB, De Feo P, De Cosmo S, Perriello G, Ventura MM, Calcinaro F, Lolli C, Campbell P, Brunetti P, Gerich JE (1984) Demonstration of a dawn phenomenon in normal human volunteers. Diabetes 33:1150-1153.

Boudaba C, Szabo K, Tasker JG (1996) Physiological mapping of local inhibitory inputs to the hypothalamic paraventricular nucleus. J Neurosci 16:7151-7160.

Buijs RM, Markman M, Nunes-Cardoso B, Hou Y-X, Shinn S (1993) Projections of the suprachiasmatic nucleus to stress-related areas in the rat hypothalamus: a light and electron microscopic study. J Comp Neurol 335:42-54.

Buijs RM, Chun SJ, Niijima A, Romijn HJ, Nagai K (2001) Parasympathetic and sympathetic control of the pancreas: a role for the suprachiasmatic nucleus and other hypothalamic centers that are involved in the regulation of food intake. J Comp Neurol 431:405-423.

Buijs RM, la Fleur SE, Wortel J, Van Heyningen C, Zuiddam L, Mettenleiter TC, Kalsbeek A, Nagai K, Niijima A (2003) The suprachiasmatic nucleus balances sympathetic and parasympathetic output to peripheral organs through separate preautonomic neurons. J Comp Neurol 464:36-48.

Card JP (2001) Pseudorabies virus and the functional architecture of the circadian timing system. J Biol Rhythms 15:453-461.

Chen QH, Haywood JR, Toney GM (2003) Sympathoexcitation by PVNinjected bicuculline requires activation of excitatory amino acid receptors. Hypertension 42:725-731.

Choi SJ, Dallman MF (1999) Hypothalamic obesity: multiple routes mediated by loss of function in medial cell groups. Endocrinology 140:4081-4088.

Cole RAL, Sawchenko PE (2002) Neurotransmitter regulation of cellular activation and neuropeptide gene expression in the paraventricular nucleus of the hypothalamus. J Neurosci 22:959-969.

Corssmit EP, Romijn JA, Sauerwein HP (2001) Regulation of glucose production with special attention to nonclassical regulatory mechanisms: a review. Metabolism 50:742-755.

Csaki A, Kocsis K, Halasz B, Kiss J (2000) Localization of glutamatergic/ aspartatergic neurons projecting to the hypothalamic paraventricular nucleus studied by retrograde transport of $\left[{ }^{3} \mathrm{H}\right] \mathrm{D}$-aspartate autoradiography. Neuroscience 101:637-655.

Cui LN, Coderre E, Renaud LP (2001) Glutamate and GABA mediate suprachiasmatic nucleus inputs to spinal-projecting paraventricular neurons. Am J Physiol 281:R1283-R1289.

Decavel C, Van Den Pol AN (1990) GABA: a dominant neurotransmitter in the hypothalamus. J Comp Neurol 302:1019-1037.

Drijfhout WJ, Kemper RHA, Meerlo P, Koolhaas JM, Grol CJ, Westerink BHC (1995) A telemetry study on the chronic effects of microdialysis probe implantation on the activity pattern and temperature rhythm of the rat. J Neurosci Methods 61:191-196.

Ferreira ML, Marubayashi U, Coimbra CC (1999) The medial preoptic area modulates the increase in plasma glucose and free fatty acid mobilization induced by acute cold exposure. Brain Res Bull 49:189-193.

Fujii T, Inoue S, Nagai K, Nakagawa H (1989) Involvement of adrenergic mechanism in hyperglycemia due to SCN stimulation. Horm Metab Res 21:643-645.

Gunion MW, Taché Y, Rosenthal MJ, Miller S, Butler B, Zib B (1989) Bombesin microinfusion into the rat hypothalamic paraventricular nu- 
cleus increases blood glucose, free fatty acids and corticosterone. Brain Res 478:47-58.

Hems DA, Rodrigues LM, Whitton PD (1978) Rapid stimulation by vasopressin, oxytocin and angiotensin II of glycogen degradation in hepatocyte suspensions. Biochem J 172:311-317.

Hermes MLHJ, Coderre EM, Buijs RM, Renaud LP (1996) GABA and glutamate mediate rapid neurotransmission from suprachiasmatic nucleus to hypothalamic paraventricular nucleus in rat. J Physiol (Lond) 496:749-757.

Hermes MLHJ, Ruijter JM, Klop A, Buijs RM, Renaud LP (2000) Vasopressin increases GABAergic inhibition of rat hypothalamic paraventricular nucleus neurons in vitro. J Neurophysiol 83:705-711.

Ionescu E, Coimbra CC, Walker CD, Jeanrenaud B (1989) Paraventricular nucleus modulation of glycemia and insulinemia in freely moving lean rats. Am J Physiol 257:R1370-R1376.

Ishikawa K, Shimazu T (1976) Daily rhythms of glycogen synthetase and phosphorylase activities in rat liver: influence of food and light. Life Sci 19:1873-1878.

Jansen ASP, Hoffman JL, Loewy AD (1997) CNS sites involved in sympathetic and parasympathetic control of the pancreas: a viral tracing study. Brain Res 766:29-38.

Kalsbeek A, Van Heerikhuize JJ, Wortel J, Buijs RM (1996) A diurnal rhythm of stimulatory input to the hypothalamo-pituitary-adrenal system as revealed by timed intrahypothalamic administration of the vasopressin V1 antagonist. J Neurosci 16:5555-5565.

Kalsbeek A, Cutrera RA, Van Heerikhuize JJ, Van Der Vliet J, Buijs RM (1999) GABA release from SCN terminals is necessary for the lightinduced inhibition of nocturnal melatonin release in the rat. Neuroscience 91:453-461.

Kalsbeek A, Garidou ML, Palm IF, Van Der Vliet J, Simonneaux V, Pévet P, Buijs RM (2000) Melatonin sees the light: blocking GABA-ergic transmission in the paraventricular nucleus induces daytime secretion of melatonin. Eur J Neurosci 12:3146-3154.

Kamohara S, Burcelin R, Halaas JL, Friedman JM, Charron MJ (1997) Acute stimulation of glucose metabolism in mice by leptin treatment. Nature 389:374-377.

Kenney MJ, Weiss ML, Patel KP, Wang Y, Fels RJ (2001) Paraventricular nucleus bicuculline alters frequency components of sympathetic nerve discharge bursts. Am J Physiol 281:H1233-H1241.

La Fleur SE (2003) Daily rhythms in glucose metabolism: suprachiasmatic nucleus output to peripheral tissue. J Neuroendocrinol 15:315-322.

La Fleur SE, Kalsbeek A, Wortel J, Buijs RM (1999) An SCN generated rhythm in basal glucose levels. J Neuroendocrinol 11:643-652.

La Fleur SE, Kalsbeek A, Wortel J, Buijs RM (2000) Polysynaptic neural pathways between the hypothalamus, including the suprachiasmatic nucleus, and the liver. Brain Res 871:50-56.

La Fleur SE, Kalsbeek A, Wortel J, Fekkes ML, Buijs RM (2001) A daily rhythm in glucose tolerance. A role for the suprachiasmatic nucleus. Diabetes 50:1237-1243.

Leibowitz SF, Sladek C, Tempel D (1988) Neuropeptide Y, epinephrine and norepinephrine in the paraventricular nucleus: stimulation of feeding and the release of corticosterone, vasopressin and glucose. Brain Res Bull 21:905-912.

Ludwig M, Leng G (1997) Autoinhibition of supraoptic nucleus vasopressin neurons in vivo: a combined retrodialysis/electrophysiological study in rats. Eur J Neurosci 9:2532-2540.

Luiten PGM, Room P (1980) Interrelations between lateral, dorsomedial and ventromedial hypothalamic nuclei in the rat. An HRP study. Brain Res 190:321-332.

Magni F, Carobi C (1983) The afferent and preganglionic parasympathetic innervation of the rat liver, demonstrated by the retrograde transport of horseradish peroxidase. J Auton Nerv Syst 8:237-260.

Martin DS, Segura T, Haywood JR (1991) Cardiovascular responses to bicuculline in the paraventricular nucleus of the rat. Hypertension 18:48-55.
Minokoshi Y, Haque MS, Shimazu T (1999) Microinjection of leptin into the ventromedial hypothalamus increases glucose uptake in peripheral tissues in rats. Diabetes 48:287-291.

Nagai K, Fujii T, Inoue S, Takamura Y, Nakagawa H (1988) Electrical stimulation of the suprachiasmatic nucleus of the hypothalamus causes hyperglycemia. Horm Metabol Res 20:37-39.

Narita K, Nishihara M, Takahashi M (1994) Concomitant regulation of running activity and metabolic change by the ventromedial nucleus of the hypothalamus. Brain Res 642:290-296.

Nonogaki K (2000) New insights into sympathetic regulation of glucose and fat metabolism. Diabetologia 43:533-549.

Nonogaki K, Iguchi A, Sakamoto N (1994) Bicuculline methiodide influences the central nervous system to produce hyperglycemia in rats. J Neuroendocrinol 6:443-446.

Obici S, Zhang BB, Karkanias G, Rossetti L (2002a) Hypothalamic insulin signaling is required for inhibition of glucose production. Nat Med 8:1376-1382.

Obici S, Feng Z, Morgan K, Stein D, Karkanias G, Rossetti L (2002b) Central administration of oleic acid inhibits glucose production and food intake. Diabetes 51:271-275.

Okamura H, Abitbol M, Julien JF, Dumas S, Berod A, Geffard M, Kitahama K, Bobillier P, Mallet J, Wiklund L (1990) Neurons containing messenger RNA encoding glutamate decarboxylase in rat hypothalamus demonstrated by in situ hybridization, with special emphasis on cell groups in medial preoptic area, anterior hypothalamic area and dorsomedial hypothalamic nucleus. Neuroscience 39:675-699.

Perreau-Lenz S, Kalsbeek A, Pevet P, Buijs RM (2004) Glutamatergic clock output stimulates melatonin synthesis at night. Eur J Neurosci 19:318-324.

Perseghin G, Regalia E, Battezzati A, Vergani S, Pulvirenti A, Terruzzi I, Baratti D, Bozzetti F, Mazzaferro V, Luzi L (1997) Regulation of glucose homeostasis in humans with denervated livers. J Clin Invest 100:931-941.

Roland BL, Sawchenko PE (1993) Local origins of some GABAergic projections to the paraventricular and supraoptic nuclei of the hypothalamus in the rat. J Comp Neurol 332:123-143.

Saper CB, Loewy AD, Swanson LW, Cowan WM (1976) Direct hypothalamo-autonomic connections. Brain Res 117:305-312.

Shimazu T (1967) Glycogen synthetase activity in liver: regulation by the autonomic nerves. Science 156:1256-1257.

Steffens AB (1969) A method for frequent sampling blood and continuous infusion of fluids in the rat without disturbing the animal. Physiol Behav 4:833-836.

Teclemariam-Mesbah R, Kalsbeek A, Pévet P, Buijs RM (1997) Direct vasoactive intestinal polypeptide-containing projection from the suprachiasmatic nucleus to spinal projecting hypothalamic paraventricular neurons. Brain Res 748:71-76.

Ueyama T, Krout KE, Van Nguyen X, Karpitskiy V, Koller A, Mettenleiter TC, Loewy AD (1999) Suprachiasmatic nucleus: a central autonomic clock. Nat Neurosci 2:1051-1053.

Van Cauter E, Polonsky KS, Scheen AJ (1997) Roles of circadian rhythmicity and sleep in human glucose regulation. Endocr Rev 18:716-738.

Vrang N, Mikkelsen JD, Larsen PJ (1997) Direct link from the suprachiasmatic nucleus to hypothalamic neurons projecting to the spinal cord: a combined tracing study using cholera toxin subunit B and Phaseolus vulgaris-leucoagglutinin. Brain Res Bull 44:671-680.

Wang D, Cui LN, Renaud LP (2003) Pre- and postsynaptic GABA $A_{B}$ receptors modulate rapid neurotransmission from suprachiasmatic nucleus to parvocellular hypothalamic paraventricular nucleus neurons. Neuroscience 118:49-58.

Watts AG, Swanson LW, Sanchez-Watts G (1987) Efferent projections of the suprachiasmatic nucleus. I. Studies using anterograde transport of Phaseolus vulgaris leucoagglutinin in the rat. J Comp Neurol 258:204-229.

Zaia CTBV, Gaziri LCJ, Zaia DAM, Delattre E, Dolnikoff MS, Timo-Iaria C (1997) Effect of chemical stimulation of the dorsomedial hypothalamic nucleus on blood plasma glucose, triglycerides and free fatty acids in rats. Brain Res Bull 42:195-198. 